TRANSACTIONS OF THE

AMERICAN MATHEMATICAL SOCIETY

Volume 355, Number 9, Pages 3699-3714

S 0002-9947(03)03357-9

Article electronically published on May 29, 2003

\title{
ON THE IWASAWA $\lambda$-INVARIANTS OF REAL ABELIAN FIELDS
}

TAKAE TSUJI

\begin{abstract}
For a prime number $p$ and a number field $k$, let $A_{\infty}$ denote the projective limit of the $p$-parts of the ideal class groups of the intermediate fields of the cyclotomic $\mathbb{Z}_{p}$-extension over $k$. It is conjectured that $A_{\infty}$ is finite if $k$ is totally real. When $p$ is an odd prime and $k$ is a real abelian field, we give a criterion for the conjecture, which is a generalization of results of Ichimura and Sumida. Furthermore, in a special case where $p$ divides the degree of $k$, we also obtain a rather simple criterion.
\end{abstract}

\section{INTRODUCTION}

Let $p$ be an odd prime number. For a number field $k$, let $k_{\infty}$ denote the cyclotomic $\mathbb{Z}_{p}$-extension of $k$ with its $n$-th layer $k_{n}(n \geq 0)$. We denote by $A_{\infty}$ the projective limit of the $p$-Sylow subgroup of the ideal class group of each $k_{n}$ with respect to the relative norms. If $k$ is a totally real number field, it is conjectured that $A_{\infty}$ is a finite abelian group ([7], [12, p. 316]), which is often called Greenberg's conjecture.

When $k$ is a real abelian field whose degree is not divisible by $p$, Ichimura and Sumida ([8], [9], [10]) discovered a good method for verifying the conjecture. In this case, we can decompose $A_{\infty}$ into a direct sum of its $\chi$-parts $A_{\infty}^{\chi}$ for Dirichlet characters $\chi$ corresponding to $k$. Then they gave a necessary and sufficient condition for $A_{\infty}^{\chi}$ to be finite in terms of certain cyclotomic units and some polynomials related to the Kubota-Leopoldt $p$-adic $L$-function $L_{p}(s, \chi)$ associated to $\chi$. It is suitable for a practical computer calculation and, for example, using it they showed that the conjecture holds when $p=3$ and $k=\mathbb{Q}(\sqrt{m})$ with $1<m<10^{4}$. (Similar criteria are also obtained by [13] and [14].)

In this paper, we study the case where $k$ is a real abelian field of arbitrary degree, including the case $p \mid[k: \mathbb{Q}]$. Although we cannot necessarily decompose $A_{\infty}$ into direct summands by using characters $\chi$ in this case, the conjecture for $k$ follows from the finiteness of all (suitably defined) $\chi$-parts $A_{\infty}^{\chi}$ of $A_{\infty}$ (cf. Lemma 2.1). The finiteness of $A_{\infty}^{\chi}$ is also conjectured, and the purpose of this paper is to give some criteria for this conjecture for $\chi$ to be true.

First, we will generalize the criterion of Ichimura and Sumida to arbitrary characters, especially characters of order divisible by $p$ (Theorem 2.6). In their proof, Ichimura and Sumida ([8, 10]) essentially used a theorem of Iwasawa [11] and Gillard [6] which describes the Galois module structure of "semi-local units modulo

Received by the editors October 27, 2002.

2000 Mathematics Subject Classification. Primary 11R23.

Key words and phrases. Iwasawa theory, Greenberg's conjecture, abelian fields. 
cyclotomic units" $\mathcal{U}^{\chi} / \mathcal{C}^{\chi}$ in terms of the $p$-adic $L$-function $L_{p}(s, \chi)$. Iwasawa and Gillard dealt with only the case where the order of $\chi$ is not divisible by $p$, and this is a main reason why Ichimura and Sumida imposed the same assumption. The author [18] removed the assumption on the order of $\chi$ in the theorem of Iwasawa and Gillard and determined the structure of $\mathcal{U}^{\chi} / \mathcal{C}^{\chi}$ for arbitrary $\chi$ (Fact 2). By using this result, we can prove our theorem by the same way as the proof of Ichimura and Sumida. When the order of $\chi$ is $p$ and $\chi(p)=1$, Fukuda and Komatsu [5] obtained a similar criterion.

In a special case where the order of $\chi$ is divisible by $p$, we also obtain the following simple sufficient condition for the conjecture to be true:

Theorem (Corollary 2.4). Assume that $\chi \omega^{-1}(p)$ is a nontrivial p-power root of unity, where $\omega$ is the Teichmüller character. If the distinguished polynomial $P_{\chi}(T)$ associated to the p-adic L-function $L_{p}(s, \chi)$ is irreducible, then $A_{\infty}^{\chi}$ is finite.

The assumption in the above theorem is satisfied only when the order of $\chi$ is divisible by $p$, since the order of $\omega$ is prime to $p$. In particular, this case was not dealt with in the papers [8], [9] and [10] of Ichimura and Sumida. We will prove the above theorem by using the fact proved in [18] that $\mathcal{U}^{\chi} / \mathcal{C}^{\chi}$ is not necessarily a cyclic module.

We remark that there indeed exist characters $\chi$ satisfying the condition in the above theorem. We give some numerical examples in $\$ 6$ We also verify in $\$ 6$ the conjecture for several real abelian fields by applying our theorem, a generalized criterion of Ichimura and Sumida, to some characters $\chi$.

\section{MAin RESUlts}

We fix an odd prime number $p$. Let $\chi$ be a nontrivial $\overline{\mathbb{Q}_{p}}$-valued even Dirichlet character of the first kind, i.e., the conductor of $\chi$ is not divisible by $p^{2}$, and let $k=k_{\chi}$ be the fixed field of the kernel of $\chi$. We denote by $k_{\infty}$ the cyclotomic $\mathbb{Z}_{p^{-}}$ extension of $k$ with its $n$-th layer $k_{n}(n \geq 0)$. We write $A_{n}=A_{k_{n}}$ for the $p$-Sylow subgroup of the ideal class group of $k_{n}$ and put $A_{\infty}=A_{k_{\infty}}:=\lim _{\longleftarrow} A_{n}$, the projective limit being taken with respect to the relative norms. We put $\Delta:=\operatorname{Gal}(k / \mathbb{Q})$ and $\Gamma:=\operatorname{Gal}\left(k_{\infty} / k\right) ;$ so $\operatorname{Gal}\left(k_{\infty} / \mathbb{Q}\right)=\Delta \times \Gamma$, since $\chi$ is of the first kind. Then we regard $A_{\infty}$ as a module over the completed group ring $\mathbb{Z}_{p}[\Delta] \llbracket \Gamma \rrbracket$. It is well known that $A_{\infty}$ is finitely generated and torsion over $\mathbb{Z}_{p}[\Delta] \llbracket \Gamma \rrbracket([12$, Theorem 5$])$. Let $\mathcal{O}$ denote the ring generated by the values of $\chi$ over $\mathbb{Z}_{p}$. For any $\mathbb{Z}_{p}[\Delta]$-module $M$, we define an $\mathcal{O}$-module $M^{\chi}$, the $\chi$-part of $M$, by

$$
M^{\chi}:=\left\{m \in M \otimes_{\mathbb{Z}_{p}} \mathcal{O} \mid \delta m=\chi(\delta) m \forall \delta \in \Delta\right\}
$$

(for the properties of the $\chi$-part, cf. e.g. [17, §II.1] and [18, §2]). If $\chi^{\prime}$ is a $\mathbb{Q}_{p^{-}}$ conjugate of $\chi$, then $M^{\chi^{\prime}} \cong M^{\chi}$. Then $\mathcal{O}$-modules $A_{n}^{\chi}$ are defined $(0 \leq n \leq \infty)$ and $A_{\infty}^{\chi}=\lim _{n} A_{n}^{\chi}$ becomes an $\mathcal{O} \llbracket \Gamma \rrbracket$-module. Let $f$ be the prime-to- $p$ part of the conductor of $\chi$ and put $q=f p$. Denote by $\boldsymbol{\mu}_{p^{n}}$ the group of $p^{n}$-th roots of unity for $n \geq 0$ and $\boldsymbol{\mu}_{p^{\infty}}:=\bigcup_{n} \boldsymbol{\mu}_{p^{n}}$. Identifying $\Gamma$ with $\operatorname{Gal}\left(k\left(\boldsymbol{\mu}_{p^{\infty}}\right) / k\left(\boldsymbol{\mu}_{p}\right)\right)$ in a natural way, we choose and fix the topological generator $\gamma$ of $\Gamma$ such that $\zeta^{\gamma}=\zeta^{1+q}$ for all $\zeta \in \boldsymbol{\mu}_{p^{\infty}}$. We identify, as usual, the completed group ring $\mathcal{O} \llbracket \Gamma \rrbracket$ with the power series ring $\Lambda:=\mathcal{O} \llbracket T \rrbracket$ by $\gamma=1+T$. Thus, $A_{\infty}^{\chi}$ is regarded as a module over $\Lambda$, and is finitely generated and torsion over $\Lambda$. For a finitely generated torsion $\Lambda$-module $M$, denote by $\operatorname{char}_{\Lambda} M$ the characteristic polynomial of $M$, which is a uniquely 
determined distinguished polynomial times a power of a fixed prime element of $\mathcal{O}$. We denote by $\lambda_{\chi}$ (resp. $\mu_{\chi}$ ) the $\lambda$-invariant (resp. $\mu$-invariant) of $A_{\infty}^{\chi}$. We know that $\mu_{\chi}=0$ by the theorem of Ferrero and Washington [4]. Greenberg's conjecture for $\chi$ is as follows:

Conjecture. Let $\chi$ be an even Dirichlet character of the first kind. It is conjectured that $A_{\infty}^{\chi}$ is finite, that is, $\operatorname{char}_{\Lambda} A_{\infty}^{\chi}=1$ or $\lambda_{\chi}=0$.

Greenberg's conjecture for a real abelian field implies the above. Indeed, by using the theorem of Ferrero and Washington [4], one can show the following:

Lemma 2.1. Let $K$ be a real abelian field. Then $A_{K_{\infty}}$ is finite if and only if $\lambda_{\chi}=0$ for all representatives of $\mathbb{Q}_{p}$-conjugate classes of Dirichlet characters $\chi$ of the first kind with $k_{\chi} \subset K_{\infty}$.

In our main results of this paper, we give two criteria for the conjecture for $\chi$. To state these, we need to recall the relation between $\operatorname{char}_{\Lambda} A_{\infty}^{\chi}$ and the KubotaLeopoldt $p$-adic $L$-function $L_{p}(s, \chi)$ associated to $\chi$. By Iwasawa, there exists a unique power series $g_{\chi}(T)$ in $\mathcal{O} \llbracket T \rrbracket$ such that

$$
g_{\chi}\left((1+q)^{s}-1\right)=L_{p}(1-s, \chi)
$$

(cf. [20, Theorem 7.10]). Using the $p$-adic Weierstrass preparation theorem and the theorem of Ferrero and Washington [4], one can uniquely write

$$
g_{\chi}(T)=u_{\chi}(T) P_{\chi}(T)
$$

for a distinguished polynomial $P_{\chi}$ in $\mathcal{O}[T]$ and a unit $u_{\chi}(T)$ of $\Lambda$. Put $\lambda_{\chi}^{*}=$ $\operatorname{deg} P_{\chi}(T)$. It follows from the Iwasawa main conjecture proved in [16] that

$$
\operatorname{char}_{\Lambda} A_{\infty}^{\chi} \mid P_{\chi}(T),
$$

and hence $\lambda_{\chi} \leq \lambda_{\chi}^{*}$ (see (3.2) and Fact 1 in $\$ 3$ ). Therefore we have $\operatorname{char}_{\Lambda} A_{\infty}^{\chi}=1$ if and only if $P(T) \nmid \operatorname{char}_{\Lambda} A_{\infty}^{\chi}$ for all distinguished irreducible factors $P(T)$ of $P_{\chi}(T)$.

One of our main theorems of this paper is as follows:

Definition. For a distinguished polynomial $P(T)$ in $\mathcal{O}[T]$ such that $P(T) \mid P_{\chi}(T)$, we define a condition $(\mathcal{T})$ as follows:

$$
v_{\mathcal{O}}(Q(q))<v_{\mathcal{O}}\left(1-\chi \omega^{-1}(p)\right) \text { where } Q(T)=P_{\chi}(T) / P(T),
$$

where $v_{\mathcal{O}}$ denotes an additive valuation of $\mathcal{O}$ and $\omega$ the Teichmüller character.

Then we have

Theorem 2.2. Let $P(T)$ be a distinguished polynomial in $\mathcal{O}[T]$ such that $P(T) \mid$ $P_{\chi}(T)$. Assume that $P(T)$ satisfies $(\mathcal{T})$. Then we have $P(T) \nmid \operatorname{char}_{\Lambda} A_{\infty}^{\chi}$.

For the condition $(\mathcal{T})$, we can easily see the following:

\section{Lemma 2.3.}

(i) If $\chi \omega^{-1}(p) \notin \boldsymbol{\mu}_{p^{\infty}}$, there exists no factor of $P_{\chi}(T)$ satisfying $(\mathcal{T})$. In particular, if the order of $\chi$ is prime to $p$ and $\chi \omega^{-1}(p) \neq 1$, there exists no factor of $P_{\chi}(T)$ satisfying $(\mathcal{T})$.

(ii) If $\chi \omega^{-1}(p) \neq 1$, a factor $P(T)$ of $P_{\chi}(T)$ satisfies $(\mathcal{T})$ if and only if

$$
v_{\mathcal{O}}(P(q))>v_{\mathcal{O}}\left(B_{1, \chi \omega^{-1}}\right)
$$

holds, where $B_{1, \chi \omega^{-1}}$ denotes the first generalized Bernoulli number. 
(iii) If $\chi \omega^{-1}(p) \in \boldsymbol{\mu}_{p^{\infty}}$, then $P_{\chi}(T)$ satisfies $(\mathcal{T})$.

(iv) ([3] Proposition 2]) If $\chi \omega^{-1}(p)=1$, then $T-q$ divides $P_{\chi}(T)$ and is the unique irreducible polynomial satisfying $(\mathcal{T})$.

In the case where $\chi \omega^{-1}(p)=1$, by (iv) of the above lemma, Theorem 2.2 just asserts that

$$
T-q \mid P_{\chi}(T) \text { and } T-q \nmid \operatorname{char}_{\Lambda} A_{\infty}^{\chi} .
$$

This is already proved under the assumption that the order of $\chi$ is prime to $p$ (9] Remark 5]). For other cases, we obtain the following.

Corollary 2.4. Assume that $\chi \omega^{-1}(p) \in \boldsymbol{\mu}_{p^{\infty}} \backslash\{1\}$. Then $P_{\chi}(T) \nmid \operatorname{char}_{\Lambda} A_{\infty}^{\chi}$. Furthermore, if $P_{\chi}(T)$ is irreducible, or if $v_{\mathcal{O}}\left(B_{1, \chi \omega^{-1}}\right)=0$, we have $\lambda_{\chi}=0$.

We will give in $[6]$ some examples satisfying the conditions in the above corollary.

Remark 1. For an arbitrary even Dirichlet character $\chi$ of the first kind, we can show that $v_{\mathcal{O}}\left(B_{1, \chi \omega^{-1}}\right)=0$ implies $A_{n}^{\chi}=\{1\}$ for all $n \geq 0$ (see \$5) ).

Next, we state the other main theorem of this paper, which is a generalization of the result of Ichimura and Sumida [8, [10] to arbitrary characters. We have to prepare some notation. We fix a distinguished polynomial $P(T)$ in $\mathcal{O}[T]$ such that $P(T) \mid P_{\chi}(T)$. Put $\omega_{n}=\omega_{n}(T)=(1+T)^{p^{n}}-1$ and $\nu_{n}=\nu_{n}(T)=\omega_{n} / T$ for $n \geq 0$. By the Leopoldt conjecture for $p$ and $k_{n}$ proved in [1], $\Lambda /\left(P, \omega_{n}\right)$ and $\Lambda /\left(P, \nu_{n}\right)$ are finite abelian groups for any $n \geq 0$. We denote by $m_{P, n}$ the exponent of $\Lambda /\left(P, \omega_{n}\right)$ (resp. $\left.\Lambda /\left(P, \nu_{n}\right)\right)$ if $\chi(p) \neq 1($ resp. $\chi(p)=1)$. Then we take a polynomial $X_{P, n}(T)$ in $\mathcal{O}[T]$ satisfying

$$
X_{P, n}(T) P(T) \equiv m_{P, n} \quad \bmod \begin{cases}\omega_{n} & \text { if } \chi(p) \neq 1, \\ \nu_{n} & \text { if } \chi(p)=1 .\end{cases}
$$

This polynomial $X_{P, n}$ is uniquely determined modulo $\omega_{n}$ (resp. $\nu_{n}$ ) since $\omega_{n}$ and $P(T)$ are relatively prime. Choose an element $\widetilde{Y}_{P, n}$ in $\mathbb{Z}[\Delta][T]$ such that

$$
\widetilde{Y}_{P, n} \equiv \widetilde{X}_{P, n} \bmod m_{P, n},
$$

where $\widetilde{X}_{P, n}$ is an element of $\mathbb{Z}_{p}[\Delta][T]$ satisfying $\chi\left(\widetilde{X}_{P, n}\right)=X_{P, n}$. Here we regard $\chi$ as a $\mathbb{Z}_{p}[T]$-linear homomorphism $\mathbb{Z}_{p}[\Delta][T] \rightarrow \mathcal{O}[T]$ induced by $\chi$. Let $\Delta_{p}$ (resp. $\Delta^{\prime}$ ) denote the $p$-Sylow subgroup (resp. the prime-to- $p$ part) of $\Delta$ :

$$
\Delta=\Delta_{p} \times \Delta^{\prime} .
$$

We put $\psi=\left.\chi\right|_{\Delta^{\prime}}$. Let

$$
e_{\psi}:=\frac{1}{\# \Delta^{\prime}} \sum_{\delta \in \Delta^{\prime}} \operatorname{Tr}(\psi(\delta)) \delta^{-1}
$$

be the idempotent of $\mathbb{Z}_{p}\left[\Delta^{\prime}\right]$ corresponding to $\psi$, where $T r$ is the trace map from the field generated by the values of $\psi$ over $\mathbb{Q}_{p}$ to $\mathbb{Q}_{p}$. Let $\alpha \in \mathbb{Z}[\Delta]$ denote an element of $\Delta_{p}$ of order $p$ (resp. $\alpha=0$ ) if $\Delta_{p}$ is nontrivial (resp. trivial), and $\mathbf{e}_{\chi}=\mathbf{e}_{\chi, P, n}$ an element of $\mathbb{Z}[\Delta]$ such that

$$
\mathbf{e}_{\chi} \equiv e_{\psi}(1-\alpha) \bmod m_{P, n} .
$$

For any $m \geq 1$, we fix a primitive $m$-th root $\zeta_{m}$ of unity with the property that $\zeta_{m m^{\prime}}^{m^{\prime}}=\zeta_{m}$ for all $m^{\prime} \geq 1$. We use cyclotomic elements in $k_{n}$ defined as follows:

$$
c_{n}=N_{\mathbb{Q}\left(\zeta_{f p^{n+1}}\right) / k_{n}}\left(1-\zeta_{f p^{n+1}}\right)^{t-1},
$$


where $t$ denotes the cardinality of the residue class field of a prime ideal of $k$ over $p$. If $f \neq 1$, i.e., the conductor of $\chi$ is not equal to $p$, then the element $c_{n}$ is a (cyclotomic) unit of $k_{n}$. Since the case where $f=1$ is treated in [8], [9] and [10], we assume $f \neq 1$ in all that follows. By the identification $\gamma=1+T$, the element $\widetilde{Y}_{P, n}$ of $\mathbb{Z}[\Delta][T]$ can act on the group $k_{n}^{\times}$. For each $n \geq 0$ consider the following condition:

$$
\left(H_{P, n}\right) \quad c_{n}^{\mathbf{e}_{\chi} \widetilde{Y}_{P, n}} \notin\left(k_{n}^{\times}\right)^{m_{P, n}} .
$$

We can see that the condition $\left(H_{P, n}\right)$ does not depend on the choices of $X_{P, n}, \widetilde{X}_{P, n}$ and $\widetilde{Y}_{P, n}$. We remark that, in the case where $\chi(p)=1$, the condition $\left(H_{P, 0}\right)$ does not hold since $c_{0}=1$ and $m_{P, 0}=1$ for any $P(T)$. The following lemma can be proved in a way similar to [9, Lemma 1] by using Lemma 4.1

Lemma 2.5. The condition $\left(H_{P, n}\right)$ implies $\left(H_{P, n+1}\right)$.

Our main theorem is stated as follows:

Theorem 2.6. Let $P(T)$ be a distinguished polynomial in $\mathcal{O}[T]$ such that $P(T) \mid$ $P_{\chi}(T)$. Then we have $P(T) \nmid \operatorname{char}_{\Lambda} A_{\infty}^{\chi}$ if and only if the condition $\left(H_{P, n}\right)$ holds for some $n \geq 0$.

The above theorem coincides with theorems in [8] and [10] if the exponent of $\Delta$ divides $p-1$.

By Theorems 2.2, 2.6 and (2.2), we obtain

Corollary 2.7. We have $\lambda_{\chi}=0$ if and only if for any distinguished irreducible polynomial $P(T)$ such that $P(T) \mid P_{\chi}(T)$ and $P(T)$ does not satisfy $(\mathcal{T})$, the condition $\left(H_{P, n}\right)$ holds for some $n \geq 0$.

By the Chebotarev density theorem, we obtain the following:

Corollary 2.8. We have $\lambda_{\chi}=0$ if and only if for any distinguished irreducible factor $P(T)$ of $P_{\chi}(T)$ that does not satisfy $(\mathcal{T})$, there exist an integer $n \geq 0$ and a prime ideal $\mathfrak{l}$ of $k_{n}$ of degree one for which the condition

$$
c_{n}^{\mathbf{e}_{\chi} \tilde{Y}_{P, n}} \bmod \mathfrak{l} \notin\left((\mathbb{Z} / l \mathbb{Z})^{\times}\right)^{m_{P, n}}
$$

holds. Here $l \mathbb{Z}=\mathfrak{l} \cap \mathbb{Q}$.

\section{Preliminaries}

We first recall the Iwasawa main conjecture and show its consequence (2.2). Let $M / k_{\infty}$ be the maximal abelian $p$-extension unramified outside $p$, and let $L / k_{\infty}$ be the maximal unramified abelian $p$-extension. As usual, we consider $\operatorname{Gal}\left(M / k_{\infty}\right)$, $\operatorname{Gal}\left(L / k_{\infty}\right)$ and $\operatorname{Gal}(M / L)$ as $\mathbb{Z}_{p}[\Delta] \llbracket \Gamma \rrbracket$-modules. Let $\mathfrak{p}$ be a prime ideal of $k$ over $p$. There exists a unique prime ideal $\mathfrak{p}_{n}$ of $k_{n}$ over $\mathfrak{p}$ since $k$ is of the first kind. We denote by $U_{n, \mathfrak{p}}$ the group of principal units in the completion $k_{n, \mathfrak{p}}$ of $k_{n}$ at $\mathfrak{p}_{n}$. Put

$$
\mathcal{U}_{n}:=\prod_{\mathfrak{p} \mid p} U_{n, \mathfrak{p}}
$$

where $\mathfrak{p}$ runs over all prime ideals of $k$ over $p$. Let $E_{n}^{\prime}$ be the group of units $\epsilon$ of $k_{n}$ such that $\epsilon \equiv 1 \bmod \mathfrak{p}_{n}$ for all $\mathfrak{p}_{n} \mid p$. Let $\mathcal{E}_{n}$ be the closure of the image of $E_{n}^{\prime}$ under the diagonal map $E_{n}^{\prime} \rightarrow \mathcal{U}_{n}$. Put

$$
\mathcal{U}:=\lim _{\longleftarrow} \mathcal{U}_{n}, \quad \mathcal{E}:=\lim _{\longleftarrow} \mathcal{E}_{n}
$$


where the projective limits are taken with respect to the relative norms. We regard $\mathcal{U}$ and $\mathcal{E}$ as modules over $\mathbb{Z}_{p}[\Delta] \llbracket \Gamma \rrbracket$. By class field theory, we have the following isomorphisms of $\mathbb{Z}_{p}[\Delta] \llbracket \Gamma \rrbracket$-modules:

$$
A_{\infty} \cong \operatorname{Gal}\left(L / k_{\infty}\right), \quad \mathcal{U} / \mathcal{E} \cong \operatorname{Gal}(M / L) .
$$

(For the latter, see [9, Lemma 3].) Put

$$
\mathfrak{X}:=\operatorname{Gal}\left(M / k_{\infty}\right) .
$$

It is known that $\mathfrak{X}$ is finitely generated and torsion over $\mathbb{Z}_{p}[\Delta] \llbracket \Gamma \rrbracket([12$, Theorem 17$])$, and we further see that $\mathfrak{X}$ is finitely generated as a $\mathbb{Z}_{p}$-module by [4]. So $A_{\infty}$ and $\mathcal{U} / \mathcal{E}$ are also finitely generated over $\mathbb{Z}_{p}$. Hence we have

$$
\operatorname{char}_{\Lambda} A_{\infty}^{\chi} \cdot \operatorname{char}_{\Lambda}\left(\mathcal{U}^{\chi} / \mathcal{E}^{\chi}\right)=\operatorname{char}_{\Lambda} \mathfrak{X}^{\chi}
$$

(cf. e.g. [18, §2]). The Iwasawa main conjecture proved in [16] asserts the following:

Fact 1. The torsion $\Lambda$-module $\mathfrak{X}^{\chi}$ has the characteristic polynomial $P_{\chi}(T)$ :

$$
\operatorname{char}_{\Lambda} \mathfrak{X}^{\chi}=P_{\chi}(T) \text {. }
$$

Hence the relation (2.2), $\operatorname{char}_{\Lambda} A_{\infty}^{\chi} \mid P_{\chi}(T)$, holds. Furthermore, Greenberg's conjecture for $(p, \chi)$ is equivalent to the following:

$$
\operatorname{char}_{\Lambda}\left(\mathcal{U}^{\chi} / \mathcal{E}^{\chi}\right)=P_{\chi}(T)
$$

Next we recall results on the structures of the $\Lambda$-modules $\mathcal{U}^{\chi}$ and $\mathcal{U}^{\chi} / \mathcal{C}^{\chi}$ in [11, [6] and [18] (Fact 2), which are essentially used in the proof of our main theorems. $\left(\mathcal{C}^{\chi}\right.$ is a group of cyclotomic units defined below.) Since we are assuming $f \neq 1$, $c_{n}=N_{\mathbb{Q}\left(\zeta_{f p^{n+1}}\right) / k_{n}}\left(1-\zeta_{f p^{n+1}}\right)^{t-1}$ is a unit in $E_{n}^{\prime}$, and hence $c_{n} \in \mathcal{E}_{n}$. We regard $c_{n}$ as an element of $\mathcal{E}_{n} \otimes \mathcal{O}$. We define $\xi_{\chi} \in \mathcal{O}[\Delta]$ by

$$
\xi_{\chi}:=\sum_{\delta \in \Delta} \chi(\delta)^{-1} \delta
$$

Then $c_{n}^{\xi_{\chi}}=\sum_{\delta \in \Delta} c_{n}^{\delta} \otimes \chi(\delta)^{-1}$ is an element of $\mathcal{E}_{n}^{\chi}$. We can see that $N_{m, n}\left(c_{m}\right)=c_{n}$ for all $m \geq n \geq 0$. Then we put

$$
c_{\infty}^{\xi_{\chi}}:=\left(c_{n}^{\xi_{\chi}}\right)_{n \geq 0} \in \mathcal{U}^{\chi}=\lim _{\longleftarrow} \mathcal{U}_{n}^{\chi}
$$

and denote by $\mathcal{C}^{\chi}$ the submodule of $\mathcal{U}^{\chi}$ generated by $c_{\infty}^{\xi_{\chi}}$ over $\Lambda$.

Let $\mathbb{T}_{n}$ denote the $\mathbb{Z}_{p}$-torsion of $\mathcal{U}_{n}^{\chi}$ and put $\mathbb{T}:=\lim _{n} \mathbb{T}_{n}$, where the projective limit is taken with respect to the relative norms. Then we have

$$
\mathbb{T}_{n}^{\chi} \cong\left(\mathcal{O} /\left(p^{n+1}, 1-\chi \omega^{-1}(p)\right)\right) \otimes_{\mathbb{Z}_{p}} \mathbb{Z}_{p}(1)
$$

and hence

$$
\mathbb{T}^{\chi} \cong \begin{cases}\{1\} & \text { if } \chi \omega^{-1}(p) \neq 1, \\ \Lambda /(T-q) & \text { if } \chi \omega^{-1}(p)=1,\end{cases}
$$

where $\mathbb{Z}_{p}(1)=\lim _{\longleftarrow} \boldsymbol{\mu}_{p^{n}}$

The following fact plays an important role to prove Theorems 2.2 and 2.6 When the order of $\chi$ is not divisible by $p$, this is the theorem of Iwasawa and Gillard ([11] and [6]). 
Fact 2. ([18, Theorem 2.1, Proposition 4.2]) There is a natural $\Lambda$-homomorphism

$$
\Psi: \mathcal{U}^{\chi} \longrightarrow \Lambda
$$

for which the kernel is $\mathbb{T}^{\chi}$ and the image is $\left(T-q, 1-\chi \omega^{-1}(p)\right)$, the ideal of $\Lambda$ generated by $T-q$ and $1-\chi \omega^{-1}(p)$. Furthermore, we have

$$
\Psi\left(c_{\infty}^{\xi_{\chi}}\right)=g_{\chi}(T)
$$

Put $\mathcal{V}_{n}^{\chi}:=\bigcap_{m \geq n} N_{m, n}\left(\mathcal{U}_{m}^{\chi}\right)$, with the norm maps $N_{m, n}$ from $k_{m}$ to $k_{n}$. In the following lemma, we determine the structure of the $\Lambda$-modules $\mathcal{V}_{n}^{\chi}$ to deal with the $\Lambda$-module $\mathcal{U}_{n}^{\chi} / \mathcal{E}_{n}^{\chi}$.

\section{Lemma 3.1.}

(i) The projection $\mathcal{U}^{\chi} \rightarrow \mathcal{V}_{n}^{\chi}$ induces the following isomorphisms:

$$
\mathcal{V}_{n}^{\chi} \cong \begin{cases}\mathcal{U}^{\chi} /\left(\mathcal{U}^{\chi}\right)^{\omega_{n}} & \text { if } \chi(p) \neq 1, \\ \mathcal{U}^{\chi} /\left(\mathcal{U}^{\chi}\right)^{\nu_{n}} & \text { if } \chi(p)=1 .\end{cases}
$$

(ii) We have an isomorphism $\mathcal{U}_{n}^{\chi} / \mathcal{V}_{n}^{\chi} \cong \mathcal{O} /(1-\chi(p))$.

By this lemma, the homomorphism $\Psi$ in Fact 2 induces a $\Lambda$-homomorphism $\Psi_{n}$ as follows:

$$
\Psi_{n}: \mathcal{V}_{n}^{\chi} \longrightarrow \Lambda /\left(\vartheta_{n}^{\chi}\right)
$$

where $\vartheta_{n}^{\chi}=\omega_{n}$ (resp. $\left.\nu_{n}\right)$ if $\chi(p) \neq 1$ (resp. $\chi(p)=1$ ). We further see that the kernel of $\Psi_{n}$ is $\mathbb{T}_{n}^{\chi}$, and we can determine the cokernel by Fact 2 .

Proof. We prove this lemma in a way similar to [6, Proposition 2] by using local class field theory. Let $D$ be the decomposition group of $p$ in $\Delta$ and put $\tilde{\chi}=\left.\chi\right|_{D}$. Fix a prime ideal $\mathfrak{p}$ of $k$ over $p$. Write $U_{n}$ for $U_{n, \mathfrak{p}}$, the group of the principal units of $k_{n, \mathfrak{p}}$, for all $n \geq 0$. We regard $U:=\varliminf_{\lim } U_{n}$ and $U_{n}$ as modules over $\mathbb{Z}_{p}[D] \llbracket \Gamma \rrbracket=\mathbb{Z}_{p}[D] \llbracket T \rrbracket$ in a natural way (with $\gamma=1+T$ ). Put $V_{n}^{\tilde{\chi}}:=\bigcap_{m \geq n} N_{m, n}\left(U_{m}^{\tilde{\chi}}\right)$ with norm map $N_{m, n}$ from $k_{m, \mathfrak{p}}$ to $k_{n, \mathfrak{p}}$. Then we have $\Lambda$-isomorphisms

$$
\mathcal{U}_{n}^{\chi} \cong U_{n}^{\tilde{\chi}} \otimes_{\mathcal{O}_{D}} \mathcal{O}, \quad \mathcal{V}_{n}^{\chi} \cong V_{n}^{\tilde{\chi}} \otimes_{\mathcal{O}_{D}} \mathcal{O}
$$

(see [18, $\S 4]$ ). Here $\mathcal{O}_{D}$ denotes the ring generated by the values of $\tilde{\chi}$ over $\mathbb{Z}_{p}$.

If $\chi(p)=1$, i.e., $\widetilde{\chi}=1$, then $k_{\mathfrak{p}}=\mathbb{Q}_{p}$. In this case, the assertions (i) and (ii) follow from [6] Proposition 2], 20, Lemma 13.53] and (3.4).

We assume that $\chi(p) \neq 1$, i.e., $\widetilde{\chi} \neq 1$. Let $\widehat{X}_{n}$ be the $p$-adic completion of $k_{n, \mathfrak{p}}^{\times}$; $\widehat{X}_{n}:=\lim _{\varliminf_{m}} k_{n, \mathfrak{p}}^{\times} /\left(k_{n, \mathfrak{p}}^{\times}\right)^{p^{m}}$, and let $\widehat{X}_{\infty}:=\lim _{\longleftarrow} \widehat{X}_{n}$. Since the (local) cyclotomic extension $k_{\infty, \mathfrak{p}} / k_{\mathfrak{p}}$ is totally ramified, we have the following commutative diagram with exact rows of $\mathbb{Z}_{p}[D] \llbracket \Gamma \rrbracket$-modules:

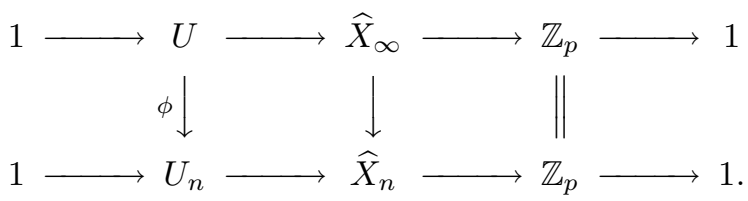

Here $D$ and $\Gamma$ act on $\mathbb{Z}_{p}$ trivially. Let $k_{n, \mathfrak{p}}^{a b}$ be the maximal abelian $p$-extension of $k_{n, \mathfrak{p}}$ for $0 \leq n \leq \infty$. We have isomorphisms $\widehat{X}_{n} \cong \operatorname{Gal}\left(k_{n, \mathfrak{p}}^{a b} / k_{n, \mathfrak{p}}\right)$ and $N \widehat{X}_{n}:=$ $\bigcap_{m \geq n} N_{m, n}\left(\widehat{X}_{m}\right) \cong \operatorname{Gal}\left(k_{n, \mathfrak{p}}^{a b} / k_{\infty, \mathfrak{p}}\right)$ of $\mathbb{Z}_{p}[D] \llbracket \Gamma \rrbracket$-modules, by local class field theory, and an isomorphism

$$
\operatorname{Gal}\left(k_{n, \mathfrak{p}}^{a b} / k_{\infty, \mathfrak{p}}\right) \cong \operatorname{Gal}\left(k_{\infty, \mathfrak{p}}^{a b} / k_{\infty, \mathfrak{p}}\right) / \omega_{n} \operatorname{Gal}\left(k_{\infty, \mathfrak{p}}^{a b} / k_{\infty, \mathfrak{p}}\right)
$$


holds. Then we have $N \widehat{X}_{n} \cong \widehat{X}_{\infty} / \widehat{X}_{\infty}^{\omega_{n}}$. Therefore the kernel of $\phi$ in (3.5) is $\widehat{X}_{\infty}^{\omega_{n}}$. So the kernel of the projection $U \tilde{\chi} \rightarrow V_{n}^{\tilde{\chi}}$ is $\left(\widehat{X}_{\infty}^{\omega_{n}}\right)^{\tilde{\chi}}$. Clearly we have $\left(\widehat{X}_{\infty}^{\omega_{n}}\right)^{\tilde{\chi}} \supset\left(\widehat{X}_{\infty}^{\tilde{\chi}}\right)^{\omega_{n}}$. Let $x^{\omega_{n}}$ be an element of $\left(\widehat{X}_{\infty}^{\omega_{n}}\right)^{\tilde{\chi}}$, i.e., $x \in \widehat{X}_{\infty} \otimes_{\mathbb{Z}_{p}} \mathcal{O}_{D}$ and $\left(x^{\omega_{n}}\right)^{\tilde{\chi}(\delta)-\delta}=1$ for all $\delta \in D$. Since $\widehat{X}_{\infty}$ has no nontrivial element that is killed by $\omega_{n}\left(\left[12\right.\right.$, Theorem 25]), we have $x^{\widetilde{\chi}(\delta)-\delta}=1$, that is, $x^{\omega_{n}} \in\left(\widehat{X}_{\infty}^{\tilde{x}}\right)^{\omega_{n}}$. Thus $\left(\widehat{X}_{\infty}^{\omega_{n}}\right)^{\tilde{\chi}}=\left(\widehat{X}_{\infty}^{\tilde{\chi}}\right)^{\omega_{n}}$. We also see that $\widehat{X}_{\infty}^{\tilde{\chi}}=U^{\tilde{\chi}}$ by (3.5) under the assumption $\widetilde{\chi} \neq 1$. Hence, the assertion (i) has been proved.

To prove (ii), we first determine the structure of $\left(N U_{n}\right)^{\tilde{\chi}} / V_{n}^{\tilde{\chi}}$, where $N U_{n}$ denotes $\bigcap_{m \geq n} N_{m, n}\left(U_{m}\right)$. If the order of $\tilde{\chi}$ is prime to $p$, the functor $(*)^{\tilde{\chi}}$ is exact. Therefore, in this case, we have $V_{n}^{\tilde{\chi}}=\left(N U_{n}\right)^{\tilde{\chi}}$, that is, $\left(N U_{n}\right)^{\tilde{\chi}} / V_{n}^{\tilde{\chi}} \cong$ $\mathcal{O}_{D} /(1-\chi(p))$. Then we consider the case where $p$ divides the order of $\tilde{\chi}$. Decompose $D=D_{p} \times D^{\prime}$ with the $p$-Sylow subgroup $D_{p}$ of $D$ and put $\widetilde{\psi}=\left.\widetilde{\chi}\right|_{D^{\prime}}$. We use the following lemma.

Lemma 3.2 (cf. [17, Lemma II.2]). Assume that $D_{p}$ is nontrivial and that $\left.\widetilde{\chi}\right|_{D_{p}}$ is a faithful character of $D_{p}$. Let $C$ be the subgroup of $D_{p}$ of order $p$ and $N_{C}$ the norm of $C$ in $\mathbb{Z}_{p}[D]$. Then, for a $\mathbb{Z}_{p}[D]$-module $M$, we have a $\mathbb{Z}_{p}[D]$-isomorphism

$$
M^{\tilde{\chi}} \cong \operatorname{ker}\left(N_{C}: e_{\widetilde{\psi}} M \rightarrow e_{\widetilde{\psi}} M\right),
$$

where $e_{\widetilde{\psi}}$ denotes the idempotent of $\mathbb{Z}_{p}\left[D^{\prime}\right]$ corresponding to $\widetilde{\psi}$.

We see that $\widehat{H}^{0}(C, U)=0$ since $k_{\mathfrak{p}} / \mathbb{Q}_{p}$ is tamely ramified. Therefore, by the above lemma and the fact that the kernel of $U \rightarrow N U_{n}$ is $\widehat{X}^{\omega_{n}}$ proved above, we have an isomorphism

$$
\left(N U_{n}\right)^{\tilde{\chi}} / V_{n}^{\tilde{\chi}} \cong \widehat{H}^{0}\left(C,\left(\widehat{X}^{e^{\tilde{\psi}}}\right)^{\omega_{n}}\right) .
$$

The fact that $\widehat{X}_{\infty}$ has no nontrivial element killed by $\omega_{n}$ implies an isomorphism $\widehat{H}^{0}\left(C,\left(\widehat{X}^{e}\right)^{\omega_{n}}\right) \cong \widehat{H}^{0}\left(C, \widehat{X}^{e_{\tilde{\psi}}}\right)$. By using $\widehat{H}^{i}(C, U)=0$ for $i=-1,0$ and (3.5), we have isomorphisms $\widehat{H}^{0}\left(C, \widehat{X}^{e} \widetilde{\psi}\right) \cong \widehat{H}^{0}\left(C, e_{\widetilde{\psi}} \mathbb{Z}_{p}\right) \cong \mathcal{O}_{D} /(1-\chi(p))$. Hence

$$
\left(N U_{n}\right)^{\tilde{\chi}} / V_{n}^{\tilde{\chi}} \cong \mathcal{O}_{D} /(1-\chi(p)) \text {. }
$$

We know that $U_{n} / N U_{n} \cong \mathbb{Z}_{p}$ (see [20, Lemma 13.53]). Thus, we have

$$
\left(N U_{n}\right)^{\tilde{\chi}}=U_{n}^{\tilde{\chi}}
$$

under the assumption $\tilde{\chi} \neq 1$. Thus, we have completed the proof.

Finally, we shall show the freeness of $\mathcal{E}^{\chi}$ (Lemma 3.5). The following is well known.

Fact 3 ([12, Theorem 18$])$. $\mathfrak{X}$ has no nontrivial finite $\mathbb{Z}_{p}[\Delta] \llbracket \Gamma \rrbracket$-submodule.

We need the following lemma, which follows from the Leopoldt conjecture for $k_{n}$ and $p$ proved in [1].

Lemma 3.3 (cf. [20, §5-5]).

(i) The inclusion $E_{n}^{\prime} \rightarrow \mathcal{E}_{n}$ induces an isomorphism

$$
E_{n}^{\prime} / E_{n}^{\prime p^{a}} \cong \mathcal{E}_{n} / \mathcal{E}_{n}^{p^{a}}
$$

for any $a \geq 0$.

(ii) $\mathcal{E}_{n}$ is $\mathbb{Z}_{p}$-torsion free. 
By the above lemma, we can regard $\mathbb{T}$ as a submodule of $\mathcal{U} / \mathcal{E}$ and also of $\mathfrak{X}$. We show the following lemma by using Fact 3

Lemma 3.4. $\mathfrak{X} / \mathbb{T}$ has no nontrivial finite $\mathbb{Z}_{p}[\Delta] \llbracket \Gamma \rrbracket$-submodule.

Proof. We identify $\mathbb{Z}_{p}[\Delta] \llbracket \Gamma \rrbracket$ with $\mathbb{Z}_{p}[\Delta] \llbracket T \rrbracket$ by $\gamma=1+T$. For a $\mathbb{Z}_{p}[\Delta] \llbracket T \rrbracket$-module $M$, we put $M_{T}:=\{m \in M \mid T m=0\}$. If $M$ is nontrivial of finite order, then $M_{T}$ is nontrivial. Thus, by Fact 3 , it suffices to show that $(\mathfrak{X} / \mathbb{T})_{T} \cong \mathfrak{X}_{T}$. Since $\mathbb{T}_{T}$ is trivial, we have an exact sequence

$$
1 \longrightarrow \mathfrak{X}_{T} \longrightarrow(\mathfrak{X} / \mathbb{T})_{T} \stackrel{\tau}{\longrightarrow}(\mathbb{T} \cap T \mathfrak{X}) / T \mathbb{T} \longrightarrow 1,
$$

where $\tau$ is induced by $x \mapsto T x$ for $x \in \mathfrak{X}$. Let $M_{0}$ be the maximal abelian extension unramified outside $p$. The restriction map $\mathfrak{X} \rightarrow \operatorname{Gal}\left(M_{0} / k\right)$ induces a surjection $\mathbb{T} \rightarrow \mathbb{T}_{0}$, and hence an isomorphism

$$
\mathbb{T} /(\mathbb{T} \cap T \mathfrak{X}) \cong \mathbb{T}_{0} .
$$

On the other hand, we see that $\mathbb{T}_{n}$ is trivial or $\boldsymbol{\mu}_{p}^{n+1} \otimes_{\mathbb{Z}_{p}} \mathbb{Z}_{p}[\Delta / D]$, where $D$ denotes the decomposition group of $p$ in $\Delta$ for all $n \geq 0$ according to whether $\mathbb{T}_{0}$ is trivial or not. Thus we have

$$
\mathbb{T} / T \mathbb{T} \cong \mathbb{T}_{0}
$$

Therefore we have $\mathbb{T} \cap T \mathfrak{X}=T \mathbb{T}$, as desired.

Using Lemma 3.4 we prove the following:

Lemma 3.5. $\Psi\left(\mathcal{E}^{\chi}\right)$ is a principal ideal generated by $\operatorname{char}_{\Lambda}\left(\mathcal{U}^{\chi} / \mathcal{E}^{\chi}\right)$. In particular, $\mathcal{E}^{\chi}$ is a free $\Lambda$-module of rank one.

Proof. There exists an element $f(T)$ of $\Lambda$ such that the principal ideal $(f(T))$ of $\Lambda$ has a submodule $\Psi\left(\mathcal{E}^{\chi}\right)$ of finite index. We first show that $f(T)$ is contained in $\Psi\left(\mathcal{U}^{\chi}\right)=\left(T-q, 1-\chi \omega^{-1}(p)\right)$. If $\chi \omega^{-1}(p) \notin \boldsymbol{\mu}_{p^{\infty}} \backslash\{1\}$, this assertion is clear since $\Psi\left(\mathcal{U}^{\chi}\right)$ is a principal ideal of $\Lambda$. Assume $\chi \omega^{-1}(p) \in \boldsymbol{\mu}_{p^{\infty}} \backslash\{1\}$. By Lemma 3.1 (i) and Fact 2, we have

$$
\bigcap_{m \geq n} N_{m, n}\left(\mathcal{E}_{m}^{\chi}\right) \cong \mathcal{E}^{\chi}\left(\mathcal{U}^{\chi}\right)^{\omega_{n}} /\left(\mathcal{U}^{\chi}\right)^{\omega_{n}} \cong\left(\Psi\left(\mathcal{E}^{\chi}\right), \omega_{n} \Psi\left(\mathcal{U}^{\chi}\right)\right) / \omega_{n} \Psi\left(\mathcal{U}^{\chi}\right) .
$$

For sufficiently large $n \geq 0, \Psi\left(\mathcal{E}^{\chi}\right) \supseteq \omega_{n}(f(T))$. Hence the above $\Lambda$-module has a finite submodule isomorphic to $\left(f(T), \Psi\left(\mathcal{U}^{\chi}\right)\right) / \Psi\left(\mathcal{U}^{\chi}\right)$. However, $\mathcal{E}_{n}^{\chi}$ is $\mathbb{Z}_{p}$-torsion free (Lemma 3.3 (ii)). Thus we have $f(T) \in \Psi\left(\mathcal{U}^{\chi}\right)$.

By Lemma 3.3 (ii), $\mathcal{E}^{\chi} \cap \mathbb{T}^{\chi}=\{1\}$. Then $\mathcal{U}^{\chi} / \mathcal{E}^{\chi}$ has a $\Lambda$-submodule isomorphic to $\mathbb{T}^{\chi}$, which we also denote by $\mathbb{T}^{\chi}$. By Fact 2 , we have an exact sequence

$$
1 \longrightarrow \mathbb{T}^{\chi} \longrightarrow \mathcal{U}^{\chi} / \mathcal{E}^{\chi} \longrightarrow \Lambda / \Psi\left(\mathcal{E}^{\chi}\right) \longrightarrow \Lambda /\left(T-q, 1-\chi \omega^{-1}(p)\right) \longrightarrow 1
$$

Then $\left(\mathcal{U}^{\chi} / \mathcal{E}^{\chi}\right) / \mathbb{T}^{\chi}$ has a finite $\Lambda$-submodule isomorphic to $(f(T)) / \Psi\left(\mathcal{E}^{\chi}\right)$, since we have proved that $f(T) \in\left(T-q, 1-\chi \omega^{-1}(p)\right)$. By (3.1), we can regard $\left(\mathcal{U}^{\chi} / \mathcal{E}^{\chi}\right) / \mathbb{T}^{\chi}$ as a submodule of $\mathfrak{X} / \mathbb{T}$. Therefore, by Lemma [3.4, we have $(f(T))=\Psi\left(\mathcal{E}^{\chi}\right)$. Furthermore, by using the above exact sequence and (3.3), we have $(f(T))=$ $\left(\operatorname{char}_{\Lambda}\left(\mathcal{U}^{\chi} / \mathcal{E}^{\chi}\right)\right)$. We have proved the lemma.

Remark 2. By using Fact 1 (the Iwasawa main conjecture), Fact 2 and the above lemma, we can see that Greenberg's conjecture for $(p, \chi)$ is equivalent to the following:

$$
\mathcal{E}^{\chi}=\mathcal{C}^{\chi}
$$




\section{Proof of the Main Results}

We rewrite the condition $\left(H_{P, n}\right)$ as follows:

Lemma 4.1. For each $n \geq 0$, the condition $\left(H_{P, n}\right)$ is equivalent to the following condition:

$\left(\mathcal{H}_{P, n}\right) \quad c_{n}^{\xi_{\chi} X_{P, n}} \notin\left(\mathcal{E}_{n}^{\chi}\right)^{m_{P, n}}$.

Proof. We fix $n \geq 0$. The isomorphism in Lemma 3.3 maps the class $\left[c_{n}^{\mathbf{e}_{\chi} \tilde{Y}_{P, n}}\right]$ in $E_{n}^{\prime} / E_{n}^{m_{P, n}}$ to the class $\left[c_{n}^{e_{\psi}(1-\alpha) \widetilde{X}_{P, n}}\right]$ in $\mathcal{E}_{n} / \mathcal{E}_{n}^{m_{P, n}}$. Thus the condition $\left(H_{P, n}\right)$ holds if and only if

$$
c_{n}^{e_{\psi}(1-\alpha) \widetilde{X}_{P, n}} \notin \mathcal{E}_{n}^{m_{P, n}} .
$$

Putting $\mathfrak{E}=\operatorname{ker}\left(\sum_{i=0}^{p-1} \alpha^{i}: \mathcal{E}_{n}^{e_{\psi}} \rightarrow \mathcal{E}_{n}^{e_{\psi}}\right)$, we have $\mathfrak{E} \mathcal{E}_{n}^{m_{P, n}}=\mathfrak{E}^{m_{P, n}}$ and $c_{n}^{e_{\psi}(1-\alpha) \widetilde{X}_{P, n}}$ $\in \mathfrak{E}$. Then the above condition holds if and only if

$$
c_{n}^{e_{\psi}(1-\alpha) \widetilde{X}_{P, n}} \notin \mathfrak{E}^{m_{P, n}} .
$$

By using Lemma 3.2, we also see that $\mathfrak{E}$ is isomorphic to $\mathcal{E}_{n}^{\chi}$ by $x^{e_{\psi}} \mapsto(x \otimes 1)^{\frac{\xi_{\chi}}{1-\alpha}}$ for $x^{e_{\psi}} \in \mathfrak{E}$. (Note that $\frac{\xi_{\chi}}{1-\alpha}$ is in $\mathcal{O}[\Delta]$.) We can see that $\xi_{\chi} \widetilde{X}_{P, n}=\xi_{\chi} X_{P, n}$. Therefore the above condition is equivalent to the condition $\left(\mathcal{H}_{P, n}\right)$.

Proof of Theorem 2.6. We shall show that $P(T) \mid \operatorname{char}_{\Lambda} A_{\infty}^{\chi}$ holds if and only if the opposite

$\left(\neg \mathcal{H}_{P, n}\right) \quad c_{n}^{\xi_{\chi} X_{P, n}} \in\left(\mathcal{E}_{n}^{\chi}\right)^{m_{P, n}}$

of $\left(\mathcal{H}_{P, n}\right)$ holds for all $n \geq 0$.

We put $Q(T)=P_{\chi}(T) / P(T)$. By Fact 1 (the Iwasawa main conjecture) and (3.2), we have

$$
\operatorname{char}_{\Lambda}\left(\mathcal{U}^{\chi} / \mathcal{E}^{\chi}\right) \cdot \operatorname{char}_{\Lambda} A_{\infty}^{\chi}=P_{\chi}(T)
$$

Then $P(T) \mid \operatorname{char}_{\Lambda} A_{\infty}^{\chi}$ if and only if $\operatorname{char}_{\Lambda}\left(\mathcal{U}^{\chi} / \mathcal{E}^{\chi}\right) \mid Q(T)$. By Lemma 3.5, the latter condition is equivalent to saying that $Q(T) \in \Psi\left(\mathcal{E}^{\chi}\right)$. Let $Q^{(n)}$ denote $Q(T) \bmod \vartheta_{n}^{\chi}$ in $\Lambda /\left(\vartheta_{n}^{\chi}\right)$. Here we recall that $\vartheta_{n}^{\chi}$ is $\omega_{n}\left(\right.$ resp. $\left.\nu_{n}\right)$ if $\chi(p) \neq 1$ (resp. $\chi(p)=1$ ). By using the $\Lambda$-homomorphism $\Psi_{n}: \mathcal{V}_{n}^{\chi} \rightarrow \Lambda /\left(\vartheta_{n}^{\chi}\right)$ defined after Lemma 3.1] we have $Q(T) \in \Psi\left(\mathcal{E}^{\chi}\right)$ if and only if

$$
Q^{(n)} \in \Psi_{n}\left(\mathcal{E}_{n}^{\chi} \cap \mathcal{V}_{n}^{\chi}\right)
$$

for all $n \geq 0$. For a fixed $n \geq 0$, it suffices to show that (4.1) is equivalent to $\left(\neg \mathcal{H}_{P, n}\right)$. Since $\Lambda /\left(\vartheta_{n}^{\chi}\right)$ is $\mathbb{Z}_{p}$-torsion free, the condition (4.1) holds if and only if

$$
m_{P, n} Q^{(n)} \in \Psi_{n}\left(\left(\mathcal{E}_{n}^{\chi} \cap \mathcal{V}_{n}^{\chi}\right)^{m_{P, n}}\right) .
$$

We defined $X_{P, n}$ to satisfy $m_{P, n} Q(T) \equiv X_{P, n}(T) P_{\chi}(T) \bmod \vartheta_{n}^{\chi}$. By Fact 2 we have $\Psi_{n}\left(c_{n}^{\xi_{\chi}}\right)=g_{\chi}(T)=u_{\chi}(T) P_{\chi}(T)$ where $u_{\chi}$ is the unit of $\Lambda$ appearing in (2.1). Hence we have

$$
u_{\chi} m_{P, n} Q^{(n)}=\Psi_{n}\left(c_{n}^{\xi_{\chi} X_{P, n}}\right) .
$$

By Fact 2 and Lemma 3.1 (i), the kernel of $\Psi_{n}$ is $\mathbb{T}_{n}$. Hence, the condition (4.2) holds if and only if

$$
c_{n}^{\xi_{\chi} X_{P, n}} \in\left(\mathcal{E}_{n}^{\chi} \cap \mathcal{V}_{n}^{\chi}\right)^{m_{P, n}} \mathbb{T}_{n}
$$


If we assume that the condition (4.4) holds, there exists $\epsilon \in \mathcal{E}_{n}^{\chi} \cap \mathcal{V}_{n}^{\chi}$ such that $\left(c_{n}^{\xi_{\chi} X_{P, n}} / \epsilon^{m_{P, n}}\right) \in \mathbb{T}_{n}$. Since $c_{n}^{\xi_{\chi}} \in \mathcal{E}_{n}^{\chi}$ and $\mathcal{E}_{n}^{\chi} \cap \mathbb{T}_{n}=\{1\}$ (Lemma 3.3 (ii)), $c_{n}^{\xi_{X} X_{P, n}}=\epsilon^{m_{P, n}}$. Thus the condition (4.4) holds if and only if

$$
c_{n}^{\xi_{\chi} X_{P, n}} \in\left(\mathcal{E}_{n}^{\chi}\right)^{m_{P, n}} \cap\left(\mathcal{V}_{n}^{\chi}\right)^{m_{P, n}} .
$$

In the case where $\chi \omega^{-1}(p) \notin \boldsymbol{\mu}_{p^{\infty}}$, we have $\Psi\left(\mathcal{U}^{\chi}\right)=\Lambda$ by Fact 2 . Then, clearly $Q(T) \in \Psi\left(\mathcal{U}^{\chi}\right)$, and hence $Q^{(n)} \in \Psi\left(\mathcal{V}_{n}^{\chi}\right)$ for all $n \geq 0$. In this case, we have $\mathbb{T}_{n}=$ $\{1\}$. Thus, by (4.3), we obtain $c_{n}^{\xi_{\chi} X_{P, n}} \in\left(\mathcal{V}_{n}^{\chi}\right)^{m_{P, n}}$. Therefore, the condition (4.5) is equivalent to $\left(\neg \mathcal{H}_{P, n}\right)$. If we assume $\chi \omega^{-1}(p) \in \boldsymbol{\mu}_{p^{\infty}}$, then $\chi(p) \notin \boldsymbol{\mu}_{p^{\infty}}$. Hence, by Lemma 3.1 (ii), we have $\mathcal{V}_{n}^{\chi}=\mathcal{U}_{n}^{\chi}$. Thus, $\left(\mathcal{E}_{n}^{\chi}\right)^{m_{P, n}} \cap\left(\mathcal{V}_{n}^{\chi}\right)^{m_{P, n}}=\left(\mathcal{E}_{n}^{\chi}\right)^{m_{P, n}}$. This completes the proof.

Proof of Theorem 2.2. The assertion follows from Theorem 2.6 and the following.

Lemma 4.2. Let $P(T)$ be a factor of $P_{\chi}(T)$. Then we have that $P(T)$ satisfies $(\mathcal{T})$ if and only if $c_{n}^{\xi_{\chi} X_{P, n}} \notin\left(\mathcal{U}_{n}^{\chi}\right)^{m_{P, n}} \mathbb{T}_{n}$ for some $n \geq 0$.

Proof. By the definition, $P(T)$ satisfies $(\mathcal{T})$ if and only if $Q(T) \notin\left(T-q, 1-\chi \omega^{-1}(p)\right)$ with $Q=P_{\chi} / P$. By Fact 2 we have $\Psi\left(\mathcal{U}^{\chi}\right)=\left(T-q, 1-\chi_{1}(p)\right)$. Hence $P(T)$ satisfies $(\mathcal{T})$ if and only if $Q(T) \notin \Psi\left(\mathcal{U}^{\chi}\right)$. Furthermore, by using an argument in the proof of Theorem 2.6 we can show that $Q(T) \notin \Psi\left(\mathcal{U}^{\chi}\right)$ holds if and only if $c_{n}^{\xi_{\chi} X_{P, n}} \notin\left(\mathcal{V}_{n}^{\chi}\right)^{m_{P, n}} \mathbb{T}_{n}$ for some $n \geq 0$. By Lemmas 2.3 (i) and 3.1 (ii), if $P(T)$ satisfies $(\mathcal{T})$, then $\mathcal{V}_{n}^{\chi}=\mathcal{U}_{n}^{\chi}$. Thus, the lemma has been proved.

Remark 3. Assume $P(T)$ satisfies $(\mathcal{T})$. Then we can directly show that $P(T) \nmid$ $\operatorname{char}_{\Lambda} A_{\infty}^{\chi}$. Indeed, we have $Q(T) \notin \Psi\left(\mathcal{U}^{\chi}\right)$ with $Q=P_{\chi} / P$ by the assumption and Fact 2 and hence $Q(T) \notin \Psi\left(\mathcal{E}^{\chi}\right)$. The latter condition is equivalent to saying that $P(T) \nmid \operatorname{char}_{\Lambda} A_{\infty}^{\chi}$ by Fact 1 , Lemma 3.5 and 3.2) (see at the beginning of the proof of Theorem 2.6).

\section{BASIC FACTS ON THE $\chi$-PARTS OF THE IDEAL ClASS GROUPS}

In this section, we shall show sufficient conditions for $A_{\infty}^{\chi}$ to be trivial (Lemma 5.1 and Remark 1), which are more or less known. We use the same notation as in the previous sections.

For a $\mathbb{Z}_{p}[\Delta]$-module $M$, we define an $\mathcal{O}$-module $M_{\chi}$, the $\chi$-quotient, by

$$
M_{\chi}:=\left(M \otimes_{\mathbb{Z}_{p}} \mathcal{O}\right) / I_{\chi}\left(M \otimes_{\mathbb{Z}_{p}} \mathcal{O}\right),
$$

where $I_{\chi}$ denotes the ideal of $\mathcal{O}[\Delta]$ generated by all elements of the form $\delta-\chi(\delta)$, $\delta \in \Delta$. If $M$ is finite, then $M^{\chi}$ and $M_{\chi}$ have the same orders (cf., e.g., [18, §2]). Thus we have

$$
\# A_{n}^{\chi}=\# A_{n, \chi} .
$$

We first show the following:

Lemma 5.1. Let $\chi$ be a (not necessarily even) Dirichlet character with $\chi(p) \notin \boldsymbol{\mu}_{p^{\infty}}$. If $A_{0}^{\chi}$ is trivial, then, for all $n \geq 0, A_{n}^{\chi}$ are trivial, and so is $A_{\infty}^{\chi}$. 
Proof. By Nakayama's lemma and (5.1), it suffices to show that

$$
A_{n, \chi} \cong A_{\infty, \chi} / \omega_{n} A_{\infty, \chi}
$$

for each $n \geq 0$. Let $L$ be the maximal unramified abelian $p$-extension of $k_{\infty}, L_{n}$ the maximal abelian extension of $k_{n}$ contained in $L$, and $H_{n}$ the Hilbert $p$-class field of $k_{n}$ for each $n \geq 0$. By class field theory, we have $A_{n} \cong \operatorname{Gal}\left(H_{n} / k_{n}\right)$ and $A_{\infty} \cong \operatorname{Gal}\left(L / k_{\infty}\right)$. We further see that $\operatorname{Gal}\left(L_{n} / k_{\infty}\right) \cong A_{\infty} / \omega_{n} A_{\infty}$, and hence $\operatorname{Gal}\left(L_{n} / k_{\infty}\right)_{\chi} \cong A_{\infty, \chi} / \omega_{n} A_{\infty, \chi}$. Then we show that $\operatorname{Gal}\left(L_{n} / k_{\infty}\right)_{\chi} \cong \operatorname{Gal}\left(H_{n} / k_{n}\right)_{\chi}$.

For a prime ideal $\mathfrak{p}$ of $k_{n}$ over $p$, let $I_{\mathfrak{p}}$ denote the inertia group of $\mathfrak{p}$ in $\operatorname{Gal}\left(L_{n} / k_{n}\right)$. Since $L_{n} / k_{n}$ is unramified outside $p$, we have an exact sequence of $\mathbb{Z}_{p}\left[\operatorname{Gal}\left(k_{n} / \mathbb{Q}\right)\right]$ modules

$$
\prod_{\mathfrak{p} \mid p} I_{\mathfrak{p}} \longrightarrow \operatorname{Gal}\left(L_{n} / k_{n}\right) \longrightarrow \operatorname{Gal}\left(H_{n} / k_{n}\right) \longrightarrow 0 .
$$

We further see that $\prod_{\mathfrak{p} \mid p} I_{\mathfrak{p}} \cong \mathbb{Z}_{p}[\Delta / D]$ (recall that $D$ is the decomposition group of $p$ in $\Delta)$. We recall that $\Delta^{\prime}$ is the prime-to- $p$ part of $\Delta, \psi=\left.\chi\right|_{\Delta^{\prime}}$ and $e_{\psi} \in \mathbb{Z}_{p}\left[\Delta^{\prime}\right]$ is its idempotent. By the assumption that $\chi(p) \notin \boldsymbol{\mu}_{p^{\infty}}$, i.e., $\psi(p) \neq 1$, we have $e_{\psi}\left(\mathbb{Z}_{p}[\Delta / D]\right)=\{1\}$, and hence $e_{\psi} \operatorname{Gal}\left(L_{n} / k_{n}\right) \cong e_{\psi} \operatorname{Gal}\left(H_{n} / k_{n}\right)$. Therefore we obtain $\operatorname{Gal}\left(L_{n} / k_{n}\right)_{\chi} \cong \operatorname{Gal}\left(H_{n} / k_{n}\right)_{\chi}$ (see [18 $\left.\left.\S 2\right]\right)$. Since $\operatorname{Gal}\left(k_{\infty} / k_{n}\right)_{\chi}=\{1\}$, we have proved the claim.

Applying Theorems 2.2 and 2.6 to verify the conjecture for $\chi$, we have only to consider the case where $\chi(p) \in \boldsymbol{\mu}_{p^{\infty}}$ or $A_{0}^{\chi}$ is nontrivial by the above lemma. We give a sufficient condition for $A_{0}^{\chi}$ to be nontrivial.

Lemma 5.2. Let $\chi$ be a Dirichlet character and $\psi$ the prime-to-p order part of $\chi$. If $e_{\psi} A_{k_{\psi}}$ is nontrivial, then so is $A_{0}^{\chi}$, where $A_{k_{\psi}}$ is the p-Sylow subgroup of the ideal class group of $k_{\psi}$.

Proof. Assume that $e_{\psi} A_{k_{\psi}}$ is nontrivial. Let $\rho$ be the Dirichlet character with $\chi=$ $\psi \rho$. Since $k_{\rho} / \mathbb{Q}$ is a cyclic extension of degree a power of $p$, at least one prime $l$ is totally ramified, and hence a prime ideal of $k_{\psi}$ above $l$ is also totally ramified in $k_{\chi}=$ $k_{\psi} k_{\rho}$. Then we see that the norm map from the $p$-Sylow subgroup of the ideal class group $A_{k_{\chi}}$ of $k_{\chi}$ to $A_{k_{\psi}}$ is surjective by class field theory. Therefore, $\left(e_{\psi} A_{k_{\chi}}\right)_{\Delta_{p}} \rightarrow$ $e_{\psi} A_{k_{\psi}}$ is also surjective; so $\left(e_{\psi} A_{k_{\chi}}\right)_{\Delta_{p}}$ is nontrivial. Let $\delta_{p}$ denote a generator of is the $p$-Sylow subgroup $\Delta_{p}$ of $\Delta$ and put $\rho=\left.\chi\right|_{\Delta_{p}}$. By the isomorphism

$$
A_{0, \chi}=\left(A_{k_{\chi}}\right)_{\chi} \cong\left(e_{\psi} A_{k_{\chi}} \otimes_{\mathcal{O}^{\prime}} \mathcal{O}\right) /\left(\delta_{p}-\rho\left(\delta_{p}\right)\right)\left(e_{\psi} A_{k_{\chi}} \otimes_{\mathcal{O}^{\prime}} \mathcal{O}\right)
$$

(cf. [18, §2]), we have

$$
\left(\left(e_{\psi} A_{k_{\chi}}\right)_{\Delta_{p}} \otimes_{\mathcal{O}^{\prime}} \mathcal{O}\right) /\left(1-\rho\left(\delta_{p}\right)\right)\left(\left(e_{\psi} A_{k_{\chi}}\right)_{\Delta_{p}} \otimes_{\mathcal{O}^{\prime}} \mathcal{O}\right) \cong A_{0, \chi} /\left(1-\rho\left(\delta_{p}\right)\right) A_{0, \chi},
$$

where $\mathcal{O}^{\prime}$ denotes the ring generated by the values of $\psi$ over $\mathbb{Z}_{p}$. Therefore, $A_{0, \chi}$ is nontrivial, as desired.

Finally we shall show that if $v_{\mathcal{O}}\left(B_{1, \chi \omega^{-1}}\right)=0$, then $A_{n}^{\chi}$ are trivial for all $n \geq 0$ (Remark 1). Let $k_{\omega \chi^{-1}}$ denote the fixed field of the kernel of the odd Dirichlet character $\omega \chi^{-1}$; so $\omega \chi^{-1}$ is a character of $\operatorname{Gal}\left(k_{\omega \chi^{-1}} / \mathbb{Q}\right)$. The $p$-Sylow subgroup of the ideal class group of the $n$-layer $k_{\omega \chi^{-1}, n}$ of the cyclotomic $\mathbb{Z}_{p^{-}}$-extension $k_{\omega \chi^{-1}, \infty} / k_{\omega \chi^{-1}}$ is a $\mathbb{Z}_{p}\left[\operatorname{Gal}\left(k_{\omega \chi^{-1}} / \mathbb{Q}\right)\right]$-module and we define $A_{n}^{\omega \chi^{-1}}$ by its $\omega \chi^{-1}$ part for each $n \geq 0$. As a consequence of the Iwasawa main conjecture proved in [16], the following was proved. 
Theorem 5.3 (16] Theorem 2 in $\$ 1.10]$, [17, Theorem II.1]). Let $\chi$ be a nontrivial even Dirichlet character. Then we have

$$
\# A_{0}^{\omega \chi^{-1}}=\#\left(\mathcal{O} / B_{1, \chi \omega^{-1}} \mathcal{O}\right) .
$$

We know the following reflection theorem (cf. [20, Theorem 10.9]).

Lemma 5.4. Let $\chi$ be an even Dirichlet character. Then the order of $A_{n, \chi} / \pi A_{n, \chi}$ divides that of $A_{n}^{\omega \chi^{-1}} / \pi A_{n}^{\omega \chi^{-1}}$ for each $n \geq 0$, where $\pi$ is a prime element of $\mathcal{O}$.

Assume $v_{\mathcal{O}}\left(B_{1, \chi \omega^{-1}}\right)=0$. By the above and (5.1), we have that $A_{0}^{\omega \chi^{-1}}$ and $A_{0}^{\chi}$ are trivial. For any character $\chi$, we have $\chi \omega^{-1}(p) \notin \boldsymbol{\mu}_{p^{\infty}}$ or $\chi(p) \notin \boldsymbol{\mu}_{p^{\infty}}$. Then, by Lemma 5.1 $A_{n}^{\omega \chi^{-1}}$ or $A_{n}^{\chi}$ are trivial for all $n \geq 0$. In the former case, the claim follows from Lemma 5.4 .

\section{ExAmples}

In this section, we verify Greenberg's conjecture for several examples by using Corollary 2.4 and Theorem 2.6. The computations in this section was carried out by Kazuo Matsuno whom the author wants to thank.

Corollary 2.4 asserts that the condition that $P_{\chi}(T)$ is irreducible or that $v_{\mathcal{O}}\left(B_{1, \chi \omega^{-1}}\right)=0$ implies $\lambda_{\chi}=0$ when $\chi \omega^{-1}(p) \in \boldsymbol{\mu}_{p^{\infty}} \backslash\{1\}$. We give some examples satisfying the condition.

The above condition is clearly satisfied when $\lambda_{\chi}^{*}=\operatorname{deg} P_{\chi}(T)=1$. In [19] Lemmas 3 and 4], the author showed that, for each even Dirichlet character $\psi$ of the first kind, there exist infinitely many characters $\rho$ of $p$-power order such that $\lambda_{\psi \rho}^{*}=\lambda_{\psi}^{*}$ and $\rho(p) \neq 1$. Then $\chi=\psi \rho$ satisfies the condition in Corollary 2.4 if $\lambda_{\psi}^{*}=$ 1 and $\psi \omega^{-1}(p)=1$. For example, these conditions are satisfied if $p=3$ and $\psi$ is the quadratic character of conductor 33 (cf., e.g., 2] for other examples). Therefore there exist infinitely many characters $\chi$ satisfying the condition in Corollary 2.4 and hence $\lambda_{\chi}=0$. In [18, a method for finding infinitely many $\chi$ 's satisfying $v_{\mathcal{O}}\left(B_{1, \chi \omega^{-1}}\right)=0$ and $\chi \omega^{-1}(p) \in \boldsymbol{\mu}_{p^{\infty}} \backslash\{1\}$ was also given. However, for such characters $\chi$, we can also verify that $\lambda_{\chi}=0$ by Lemma 5.1 instead of Corollary 2.4 since we see that $A_{0}^{\chi}=0$ if $\lambda_{\chi}^{*}=1$ or $v_{\mathcal{O}}\left(B_{1, \chi \omega^{-1}}\right)=0$ (for the latter see 95 ). So we next give examples such that $A_{0}^{\chi}$ is nontrivial and $P_{\chi}(T)$ is irreducible.

Let $p=3$ and $\psi$ be the quadratic character of conductor $321=3 \cdot 107$. For a character $\rho$ of order 3 , we put $\chi=\psi \rho$. We see that $e_{\psi} A_{k_{\psi}}=A_{\mathbb{Q}(\sqrt{321})}$ is nontrivial (the class number of $\mathbb{Q}(\sqrt{321})$ is 3 ), and hence $A_{0}^{\chi}$ is also nontrivial by Lemma 5.2. Let $\rho$ be a character of order 3 whose conductor is 7 . Then $\rho(3) \neq 1$; so $\chi \omega^{-1}(3) \in \boldsymbol{\mu}_{3} \backslash\{1\}$. For this $\chi$, we calculate $P_{\chi}(T)$ modulo a power of $p$ as in [10] and [5] by using the fact that the Stickelberger elements produce $g_{\chi}(T)$ and check the irreducibility of $P_{\chi}(T)$. More precisely, we calculate $N P_{\chi}(T)=$ $P_{\chi}(T) P_{\chi^{-1}}(T) \in \mathbb{Z}_{3}[T]\left(\chi^{-1}\right.$ is a $\mathbb{Q}_{3}$-conjugate of $\left.\chi\right)$, since it is easier to handle $\mathbb{Z}_{3}$-coefficient polynomials than $\mathcal{O}=\mathbb{Z}_{3}\left[\zeta_{3}\right]$-coefficient ones. We have

$$
N P_{\chi}(T) \equiv T^{4}+5130 T^{3}+1020 T^{2}+2214 T+4977 \bmod 3^{8} .
$$

We see that $N P_{\chi}(T)$ is irreducible over $\mathbb{Z}_{3}$. So $P_{\chi}(T)$ is also irreducible over $\mathcal{O}$. Thus, this $\chi$ satisfies the condition in Corollary 2.4 and we have $\lambda_{\chi}=0$. Moreover, this implies that Greenberg's conjecture for $k_{\chi}$, the cyclic field of degree 6 whose conductor is $2247=3 \cdot 7 \cdot 107$, is true by Lemma 2.1 In fact, Ichimura and Sumida 
[10, Proposition] showed that $\lambda_{\psi}=0$, and we can see that $\lambda_{\rho}=0$ by Iwasawa's lemma (cf. [20, Lemma 10.4]) since $\rho(3) \neq 1$.

We further check the irreducibility of $P_{\psi \rho}(T)$ for a nontrivial character $\rho$ of cyclic cubic fields $k_{\rho}$ with conductor less than $10^{3}$. There are 128 such fields, and 86 fields among them satisfy $\rho(3) \neq 1$ (i.e., 3 does not decompose in those fields). Among these 86 fields, $P_{\psi \rho}(T)$ is irreducible for those $\rho$ whose conductor is in the following list:

$$
\begin{aligned}
& 7,43,97,109,127,139,157,181,211,229,277,337, \\
& 349,379,409,607,691,709,733,739,751,877,907, \\
& 217=7 \cdot 31,301=7 \cdot 43,427^{\dagger}=7 \cdot 61,469^{\dagger}=7 \cdot 67, \\
& 511^{\dagger}=7 \cdot 73,553=7 \cdot 79,721^{\dagger}=7 \cdot 103 .
\end{aligned}
$$

We remark that there are two cyclic cubic fields $k_{\rho}$ satisfying $\rho(3) \neq 1$ whose conductor is one of the numbers with $\dagger$ in the above list. (We have only one such field for others.) Among them, $P_{\psi \rho}(T)$ are irreducible for both fields of conductor $427,511,721$ and for one of the fields of conductor 469 . Thus we obtain 33 cyclic cubic fields $k_{\rho}$ of conductor less than $10^{3}$ such that $\chi=\psi \rho$ satisfies the assumption of Corollary 2.4.

We can see that $\lambda_{\rho}=0$ if the conductor of $\rho$ is prime and $\rho(3) \neq 1$ by Iwasawa's lemma. Furthermore, Yamamoto kindly informed us that we know that $\lambda_{\rho}=0$ for $\rho$ whose conductor is $217,301,427,469$ or 721 (see below for 553). Thus, for such $\rho$ 's, Greenberg's conjecture for $k_{\chi}=k_{\rho}(\sqrt{321})$ is true by Lemma 2.1 .

We apply Theorem 2.6 to several examples.

Let $p=3, k_{\chi}$ be a unique cubic field of conductor $553=7 \cdot 79$ in which 3 does not decompose and $\chi$ a nontrivial character of $k_{\chi}$. We fix a generator $\sigma$ of $\Delta_{p}(=\Delta)$ and put $\chi(\sigma)=\zeta$, a nontrivial $p$-th root of unity. We have

$$
P_{\chi}(T) \equiv T-(122+160 \zeta) \bmod 3^{5} .
$$

We check the condition $\left(H_{P_{\chi}, 2}\right)$. We see that $m_{P_{\chi}, 2}=3^{3}=27$ and

$$
\begin{aligned}
\widetilde{Y}_{P_{\chi}, 2} \equiv T^{8} & +(23+25 \zeta) T^{7}+(3+3 \zeta) T^{6}+(24+15 \zeta) T^{5} \\
& +(6+3 \zeta) T^{4}+9 \zeta T^{3}+(21+9 \zeta) T^{2}+(24+21 \zeta) T+9+18 \zeta \bmod 3^{3} .
\end{aligned}
$$

Let $l=29863$. Then a prime ideal $\mathfrak{l}$ of $k_{2}$ above $l$ is of degree one. We verify that $c_{2}^{(1-\sigma) \tilde{Y}_{P_{\chi}, 2}} \bmod \mathfrak{l} \notin(\mathbb{Z} / l \mathbb{Z})^{\times 27}$, and so we have $\lambda_{\chi}=0$. Greenberg's conjecture for $k_{\chi}$ (and $k_{\chi}(\sqrt{321})$ also) is also true.

Let $p=5$ and $\chi$ be a character of order 5 with prime conductor $f$. By a result of Kurihara [15, §4.4], we know that $\lambda_{\chi}=0$ for all $f<10^{5}$ except $f=$ $38851,41201,84551$. We consider the case $f=38851$. By computing $N P_{\chi}(T)=$ $\prod_{i=1}^{4} P_{\chi^{i}}(T) \in \mathbb{Z}_{5}[T]$, we see that $P_{\chi}(T)$ decomposes into a product of two irreducible factors $P_{1}(T)$ and $P_{2}(T)$ of degrees 1 and 3 respectively. In [15, Kurihara introduced an invariant $\kappa$ associated to $\chi$ and showed that, for a factor $P(T)$ of $P_{\chi}(T)$, we have $P(T) \nmid \operatorname{char}_{\Lambda} A_{\infty}^{\chi}$ if $\operatorname{deg} P(T) \geq \kappa$ (cf. [15, Remark 1.11]). We have $\kappa=2$ in this case. So we check the conditions in Theorem [2.6] only for the factor $P_{1}(T)$ of degree 1 . We have

$$
P_{1}(T) \equiv T-\left(1313+416 \zeta+1834 \zeta^{2}+2427 \zeta^{3}\right) \bmod 5^{5} .
$$


We check the condition $\left(H_{P_{1}, 1}\right)$. We see that $m_{P_{1}, 1}=5$ and

$$
\widetilde{Y}_{P_{1}, 1}=T^{3}+\left(3+\sigma+4 \sigma^{2}+2 \sigma^{3}\right) T^{2} .
$$

We verify that $c_{1}^{(1-\sigma) \widetilde{Y}_{P_{1}, 1}} \bmod \mathfrak{l} \notin(\mathbb{Z} / l \mathbb{Z})^{\times 5}$ with $l=9712751$, where a prime ideal $\mathfrak{l}$ of $k_{1}$ above $l$ is of degree one.

We further consider the cases where $f=41201,84551$ in the above setting. We see that $P_{\chi}(T)$ decomposes into a product of two irreducible factors $P_{1}(T)$ and $P_{2}(T)$ of degrees 1 and 3 respectively, in both cases, and have $\kappa=2$ (resp. 3) if $f=41201$ (resp. 84551). Hence we have only to check the conditions in Theorem 2.6 for the factor $P_{1}(T)$ of degree 1 for both $f$ 's. We verify that the condition $\left(H_{P_{1}, 1}\right)$ holds in each case. Therefore, together with the result of Kurihara [15, §4.4], we have that $\lambda_{\chi}=0$ for $p=5$ and all primes $f<10^{5}$.

\section{ACKNOWLEDGEMENTS}

The author would like to express her sincere gratitude to Professor Humio Ichimura and Doctor Hiroki Sumida for various advice and for encouragement. She also would like to thank Doctor Kazuo Matsuno for carrying out calculations in 96 which allowed us to verify Greenberg's conjecture for several examples, and thank Doctor Gen Yamamoto for kindly informing us about known results on the conjecture.

\section{REFERENCES}

[1] A. Brumer, On the units of algebraic number fields, Mathematika 14 (1967) 121-124. MR 36:3746

[2] D. S. Dummit, D. Ford, H. Kisilevsky and W. Sands, Computation of Iwasawa lambda invariants for imaginary quadratic fields, J. Number Theory 37 (1991) 100-121. MR 92a:11124

[3] B. Ferrero and R. Greenberg, On the behavior of $p$-adic L-functions at $s=0$, Invent. Math. 50 (1978) 91-102. MR 80f:12016

[4] B. Ferrero and L. C. Washington, The Iwasawa invariant $\mu_{p}$ vanishes for abelian number fields, Ann. of Math. (2) 109 (1979) 377-395. MR 81a:12005

[5] T. Fukuda and K. Komatsu, Ichimura-Sumida criterion for Iwasawa $\lambda$-invariants, Proc. Japan Acad. Ser. A Math. Sci. 76 (2000) 111-115. MR 2001g:11168

[6] R. Gillard, Unités cyclotomiques, unités semi-locales et $\mathbb{Z}_{\ell}$-extensions II, Ann. Inst. Fourier (Grenoble) 29 (1979) 49-79. MR 81e:12005a

[7] R. Greenberg, On the Iwasawa invariants of totally real number fields, Amer. J. Math. 98 (1976) 263-284. MR 53:5529

[8] H. Ichimura and H. Sumida, On the Iwasawa invariants of certain real abelian fields, Tôhoku Math. J. 49 (1997) 203-215. MR 98e:11128a

[9] H. Ichimura and H. Sumida, On the Iwasawa $\lambda$-invariant of the real p-cyclotomic field, J. Math. Sci. Univ. Tokyo. 3 (1996) 457-470. MR 98e:11128b

[10] H. Ichimura and H. Sumida, On the Iwasawa invariants of certain real abelian fields II, Internat. J. Math. 7 (1996) 721-744. MR 98e:11128c

[11] K. Iwasawa, On some modules in the theory of cyclotomic fields, J. Math. Soc. Japan 16 (1964) 42-82. MR 35:6646

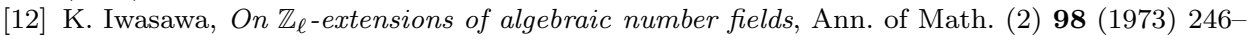
326. MR 50:2120

[13] J. Kraft and R. Schoof, Computing Iwasawa modules of real quadratic number fields, Compositio Math. 97 (1995) 135-155. MR 97b:11129

[14] M. Kurihara, The Iwasawa $\lambda$-invariants of real abelian fields and the cyclotomic elements, Tokyo J. Math. 22 (1999) 259-277. MR 2001a:11182

[15] M. Kurihara, Remarks on the $\lambda_{p}$-invariants of cyclic fields of degree $p$, preprint.

[16] B. Mazur and A. Wiles, Class fields of abelian extensions of $\mathbb{Q}$, Invent. Math. 76 (1984) 179-330. MR 85m:11069 
[17] D. Solomon, On the classgroups of imaginary abelian fields, Ann. Inst. Fourier (Grenoble) 40 (1990) 467-492. MR 92a:11133

[18] T. Tsuji, Semi-local units modulo cyclotomic units, J. Number Theory 78 (1999) 1-26. MR 2000f: 11148

[19] T. Tsuji, Greenberg's conjecture for Dirichlet characters of order divisible by $p$, Proc. Japan Acad. Ser. A Math. Sci. 77 (2001) 52-54. MR 2002d:11130

[20] L. C. Washington, Introduction to Cyclotomic Fields, Graduate Texts in Math. 83, SpringerVerlag, New York, 1982. MR 85g:11001

Department of Mathematics, Tokai University, Hiratsuka, Kanagawa, 259-1292, Japan

E-mail address: tsuji@sm.u-tokai.ac.jp 